\title{
Comunes urbanos: de la gestión colectiva al derecho a la ciudad
}

Mauro Castro-Coma. Universidad Politécnica de Catalunya (UPC), Barcelona, España. Marc Marti-Costa. Flacso-Ecuador, Quito, Ecuador.

RESUMEN | Existe un creciente interés y una emergente literatura académica en los estudios urbanos sobre los comunes y su aplicación. Por un lado, destacan aquellas aportaciones que aplican al ámbito urbano, los estudios de Elinor Ostrom sobre las formas de autogestión de los recursos naturales de uso común. Por el otro, la literatura crítica sobre el neoliberalismo, las nuevas formas de acumulación por desposesión y las resistencias a ellas en forma de movimientos sociales también han abordado los comunes en la ciudad desde diferentes perspectivas de origen marxista. En este trabajo se ordenan y se analizan las contribuciones de ambas aproximaciones, sus puntos críticos, así como sus posibles complementariedades, para avanzar hacia una mejor conceptualización y una futura agenda de investigación sobre los comunes urbanos.

PALABRAS ClAVE | teoría urbana, movimientos sociales, gestión urbana.

ABSTRACT | There is a growing interest and emerging academic literature regarding the commons and their application in urban studies. On the one hand, those contributions related to urban areas, Elinor Ostrom studies on forms of self-management of the natural common pool resources, are pointed. On the other hand, the critical literature on neoliberalism, new forms of accumulation by dispossession and the resistances to them in the form of social movements have also addressed the commons in the city from different Marxist perspectives. In this paper the contributions of both approaches are addressed and analyzed, as well as their critical points and possible complementarities to move towards a better conceptualization and a future research agenda on the urban commons.

KEY WORDS | urban theory, social movements, urban management. 


\section{Comunes y ciudad}

Desde su aplicación a los recursos naturales y posteriormente a los recursos digitales, la mirada sobre los bienes comunes se está aplicando también al fenómeno urbano. La literatura, aunque reciente, es bastante amplia y destaca por la existencia de múltiples interpretaciones sobre qué se entiende por "común"1, y su aplicación al territorio urbano y a la gestión de la ciudad. Ante esta situación, consideramos necesario realizar una reflexión que evalúe las posibilidades y límites del marco teórico de los comunes como proyecto explícitamente urbano, retomando dos de los principales caminos o "puntos de entrada" (Hess, 2008) transitados por la literatura especializada, teniendo en cuenta las numerosas conexiones transversales que existen entre ellos.

Así, como primer punto de entrada encontramos la denominada vertiente neoinstitucionalista, cuya principal exponente es la politóloga estadounidense Elinor Ostrom, corriente que pone el acento en analizar los paradigmas institucionales y las normas de gobernanza de las que se dotan las comunidades para gestionar los llamados recursos comunes (RC) o de acervo común (common-pool-resources) ${ }^{2}$. El segundo punto de entrada lo situamos dentro de la corriente marxista que vincula los comunes y su compleja interrelación con las prácticas socioeconómicas de cercamiento, centrando su atención en las múltiples formas en que el proceso está siendo tanto implementado como resistido en el ámbito urbano.

El artículo se estructura de la siguiente manera: en el primer apartado abordaremos la literatura relativa a las formas de gobernanza de los RC y las importantes diferencias que existen entre los recursos naturales que Ostrom analiza y los recursos físicos, sociales y culturales que encontramos en el ámbito urbano. En un segundo apartado ponemos el foco en aquellos autores que han vinculado las luchas por el común como respuesta a los cercamientos y a las lógicas de apropiación por desposesión en la ciudad neoliberal, enfoque muy próximo a la literatura sobre movimientos sociales urbanos y las luchas por el derecho en la ciudad. Por último, terminaremos con algunas conclusiones sobre la aportación al campo que produce el diálogo entre ambas aproximaciones.

Si bien la literatura en el ámbito de los comunes urbanos (urban commons) ha estado dominada hasta ahora por el mundo anglosajón (tal y como lo refleja la

1 Rápidamente nos podemos dar cuenta de que alrededor de los "commons" hay diversas ambigüedades y conceptos asociados que no son sinónimos entre sí, como el de riqueza común, recursos comunes, propiedad común, bien común, etcétera. De hecho, aún está en discusión cuál es la traducción de "commons" más pertinente al castellano, ya sea "común", "procomún" (sustantivo derivado de "provecho" y "común", y que significa "utilidad pública”) o "bienes comunes”. Además, es un campo de estudio donde están convergiendo muchas disciplinas diferentes (filosofía, economía, economía política, sociología, ciencia política, ecología, etcétera), lo que complica aún más la clarificación conceptual. Interesante en este sentido es mirar la compilación realizada por Hess (2008).

2 Ostrom entiende "common-pool resource" como "natural or man-made resource system that is sufficiently large as to make it costly (but not impossible) to exclude potential beneficiaries from obtaining benefits from its use". Debido a su mayor simplicidad, nosotros preferimos traducir los "common-pool resources" como "recursos comunes". 
bibliografía utilizada), es importante destacar otras realidades, como la latinoamericana, con una importante tradición en la temática de los bienes comunes y experiencias concretas de gran calado y relevancia social (véase, entre otros, a Gutiérrez, 2008; o Spronk \& Webber, 2007).

\section{La autogestión de los "recursos comunes urbanos"}

Los trabajos de Elinor Ostrom (1990) sobre la gestión de los recursos naturales han sido de gran inspiración y guía para repensar las formas de gestión colectiva de los recursos urbanos de uso común. Desde una perspectiva basada en la racionalidad individual y colectiva, Ostrom analiza las críticas, los problemas y las posibilidades de la acción colectiva -ni estatal ni privada- para una gestión eficiente de los recursos naturales. Su contribución arranca con una crítica a la metáfora de la "tragedia de los comunes" popularizada por Garret Hardin (1968), muy próxima a las aportaciones de Olson (1965) sobre la lógica de la acción colectiva. Hardin señala cómo un recurso común que es abierto a todo el mundo ${ }^{3}$, terminará por degradarse, porque cada individuo tenderá a maximizar su propio beneficio en detrimento del mantenimiento del recurso de forma colectiva, incluso cuando los beneficios de una estrategia colectiva fueran mayores.

Siguiendo tal argumentación, la única solución a esta "tragedia" sería establecer o bien un sistema de regulación basado en la propiedad privada y en el mercado, en el que cada individuo se responsabilice de su propiedad, o bien un sistema de control estatal, basado ya sea en la planificación o bien en la jerarquía. En cambio, Ostrom señala, por un lado, los costes -normalmente invisibilizados- que suponen tanto la mantención de la propiedad en calidad de privada (vallas, vigilancia, menos capacidad de rentabilizar las inversiones...) como el control, conservación y mejora continuada que también existen para una entidad externa como el Estado. Por otro lado, ilustra a través de una serie de casos concretos la viabilidad de la acción colectiva para la gestión de recursos comunes, siempre que se dé una serie de condiciones que faciliten la gobernanza colectiva de ese recurso.

\section{Recursos, comunidad y gobierno}

Si nos centramos en el ámbito urbano, se detectan importantes diferencias en relación con los ejemplos de RC autogestionados investigados por Ostrom y los recursos comunes urbanos autogestionados por las propias comunidades en las ciudades. Tal y como analiza Hess (2008) al hablar sobre la literatura referida a los "nuevos comunes" (recursos compartidos que recientemente han evolucionado o han sido reconocidos como comunes), el término ha perdido conexión con los conceptos académicos sobre los recursos comunes tradicionales (RC) y ha pasado a ser definido de forma más abierta y expansiva -y, por tanto, más difusa analíticamente (Colding \& Barthel, 2013)-, no limitada a un tipo específico de bien económico (que cumple

Hardin (1968) confunde "recurso abierto" (open access), no regulado, con "recurso de gestión común”. Por el contrario, para Ostrom (1990), el problema no es el recurso común per se, sino la gobernanza de ese recurso. 
las características de rivalidad y no exclusividad) o a un tipo de régimen de propiedad (Hess, 2008, p. 34).

\section{Recursos}

La primera cuestión es qué recursos existentes en la ciudad se pueden considerar como bienes comunes. La literatura diferencia los recursos comunes entre aquellos materiales (suelo, viviendas, infraestructuras, equipamientos, espacios públicos, espacios verdes) e inmateriales (información, conocimiento, creaciones culturales, cooperación, seguridad, etcétera); los naturales (el agua, el aire, la riqueza de la tierra) de los artificiales (es decir, sociales, culturales o saberes colectivos); universales (cuyo acceso debe ser garantizado a todos y todas) o producidos localmente (con una comunidad de referencia); abundantes y escasos, etcétera.

En un intento de concretar, Efrat Eizenberg (2012) habla de "los comunes realmente existentes" para referirse a aquellos recursos compartidos en entornos urbanos gobernados por regímenes de propiedad común; es decir, arreglos institucionales que no suponen ni la administración del Estado ni la propiedad privada, sino que están basados en la autogestión de una comunidad local. Esta autora cita como ejemplos los jardines comunitarios de Nueva York, cooperativas de trabajadores y experiencias de propiedad colectiva de vivienda destinada a poblaciones urbanas pobres y administrada por ellas. Otros autores se refieren a estos mismos ejemplos como "comunes vecinales" (Hess, 2008), destacando su capacidad de proteger, preservar, gestionar, etcétera, recursos locales a partir de comunidades próximas a ellos. De todas formas, la revisión de la literatura existente muestra una amplia variedad a la hora de hacer referencia a este tipo de recursos urbanos, cada uno con sus particularidades en relación con el régimen de propiedad y su gestión. Casos de estudio habituales son jardines y huertos comunitarios (Fernández \& Burch, 2003; Foster, 2006; 2011; Sheldon, 2010), los mercados callejeros (Parker \& Johansson, 2011), parques y otras instalaciones recreativas (Matisoff \& Noonan 2012) y espacios públicos más en general (Cooper, 2006; Kassa, 2008; Low \& Smith, 2006), casas ocupadas (Hodkinson \& Chatterton, 2006), cooperativas de viviendas (Saegert \& Benítez, 2005), o incluso las comunidades cerradas (Colding, 2011; Le Goix \& Webster, 2006; Lee \& Webster, 2006) entre otros.

También hay autores que incluyen como recursos compartidos aspectos más inmateriales, como el de la seguridad, abordando - por ejemplo- cómo la comunidad se organiza para prevenir la criminalidad en un área determinada de la ciudad (Bennett, Holloway \& Farrington, 2006; Wagenaar \& Soeparman, 2004). En una escala mayor, ha habido tentativas de conceptualizar las infraestructuras básicas que posibilitan la vida en la ciudad como comunes urbanos (Bravo \& De Moor, 2008; Frischmann, 2005; 2006). Los ejemplos van desde los sistemas de distribución de agua (Bakker, 2007; Wutich, 2009), de infraestructuras portuarias (Selsky \& Memon, 1997), de carreteras (Blomkvist \& Larsson, 2013), de electricidad (Byrne, Martínez \& Ruggero, 2009; Lambing, 2012) o de recolección y tratamiento de desechos (Cavé, 2012; Post \& Baud, 2003), los ecosistemas naturales en la ciudad (Svendsen \& Campbell, 2008) o incluso los sistemas de regulación del suelo (Porter et al., 2011; Salingaros, 2010). Debido a su importancia como facilitadores de 
la vida urbana y a la complejidad de su gestión, suele apelarse a las instituciones públicas para la provisión, cuidado y mantenimiento de estos recursos, ya sea de forma directa (Sofoulis \& Williams, 2008) o bien utilizando formas híbridas de cogestión o coproducción con la sociedad civil (McShane, 2010).

Lo anterior introduce la cuestión de la escala: existen recursos que pueden estar sujetos a formas de gestión local por parte de comunidades claramente delimitadas (y por lo general de dimensiones reducidas) y existen comunes (por ejemplo, el espacio público o las infraestructuras colectivas) que tienen como referencia comunidades mucho más amplias, que necesitan una gestión más abierta y no propietaria y que deben pensarse, por tanto, en términos de acceso incluyente y de una participación expansiva.

En suma, qué es un recurso común urbano y qué no es, constituye una cuestión abierta, contextual y sobre todo política: como veremos a continuación, un recurso común es un recurso común cuando existe una comunidad que lo reclama como tal.

\section{Comunidad}

Tal y como puso de relieve Ostrom, los comunes, más allá de ser considerados simplemente como unos recursos (el software, el agua o el espacio público) con características concretas (rivalidad en el consumo y no excluyentes en el acceso), deben venir acompañados de una comunidad activa que los gestiona, dotándose de normas compartidas. El común no debe ser interpretado a partir de sus características intrínsecas como un tipo particular de activo, cosa o recurso, sino que se define en relación con el sujeto (por tanto, como una relación social) en el contexto localizado de una comunidad. Más complicado es precisar qué entendemos por "comunidades" en el ámbito urbano.

En los casos más exitosos que describe Ostrom, las comunidades que cuidan del recurso y hacen sostenible su uso son, aun con excepciones, más bien pequeñas, con lazos fuertes y normas compartidas estables. La descripción de este tipo de comunidades resuena más a sociedades rurales y tradicionales que no a aquellas sociedades urbanitas, caracterizadas por sus múltiples asociaciones temporales entre extraños, basadas en el interés.

A menudo, el barrio se ha conceptualizado como el espacio intermedio entre lo rural y lo urbano, entre lo doméstico y la metrópoli, entre lo privado y lo institucional. Desde una perspectiva funcional, el barrio también significa la unidad mínima de reproducción social de la ciudad, un territorio donde satisfacer, a través de diferentes ámbitos de sociabilidad, recursos y equipamientos, las necesidades básicas de autoabastecimiento, socialización, educación o salud cotidiana. Ligado a este último punto, el barrio también ha sido analizado como el espacio de organización política, ya sea por las resistencias a los procesos de gentrificación (Smith, 2002), por las reivindicaciones de servicios y equipamientos urbanos (Castells, 1977) o simplemente como esfera de participación en el gobierno y en las políticas locales. En el contexto barrial así concebido, la comunidad ya no se entiende como un grupo más bien cerrado y homogéneo, sino que se resignifica como colectividades plurales pero con una serie de intereses y necesidades compartidas a partir 
de la proximidad 4 . Estos factores, como son la proximidad residencial y una cierta dependencia de determinados recursos compartidos, hacen del barrio un espacio urbano especialmente propicio para desarrollar experiencias de gestión colectiva de recursos comunes urbanos.

No obstante lo anterior, diferentes autores han señalado también la diversidad de características que puede tener este tipo de comunidades en el ámbito urbano. Pueden ser exclusivas o integradoras, regresivas o progresistas (Kohn, 2004; McCarthy, 2005); pueden referirse a un pequeńo grupo de usuarios, a un vecindario entero o a grupos que operan en el espacio digital (Blomley, 2008; Foster, 2011); y pueden ser sedentarias o temporales. En cualquier caso, son organizaciones sociales que, lejos de la idea de comunidad homogénea y de corte esencialista, se fundamentan en el hacer común, es decir, en la propia acción de gestionar y democráticamente gobernar recursos comunes.

En cualquier caso, ya se trate de comunidades de base barrial o de otro tipo, está claro que: i) aunque se generen a partir de "formas similares de propiedad colectiva, ellas tienen (tal y como sucede con la naturaleza elitista y excluyente de los condominios cerrados) efectos sociales y políticos muy diferentes" (Kohn, 2004, p. 10); ii) las debemos entender como formas mucha más abiertas, inestables y con menos dependencia del recurso común que los casos exitosos que analiza Ostrom. En los recursos comunes urbanos, normalmente la subsistencia de los "apropiadores" no depende únicamente de la explotación del recurso, lo que tiene importantes consecuencias en las motivaciones para mantener el recurso o para controlar a los otros usuarios 5 . Esto no quiere decir que los usuarios no obtengan ningún tipo de beneficio (ya sea económico, social o político), sino que el beneficio es mucho más indirecto, por lo que otras motivaciones (fundamentalmente cívicas o políticas) más allá de la propia subsistencia, deben estar presentes. Esto implica que el cálculo de costes y beneficios difícilmente se puede medir a partir de la perspectiva del homo economicus subyacente en la teoría ostromiana. El mismo Olson (1965) reconocía que su teoría basada en la racionalidad económica no funcionaba demasiado bien para estudiar las organizaciones filantrópicas, comunales y religiosas.

\section{Gobierno local}

Por último, es necesario apuntar a la relación entre los recursos comunes urbanos y el gobierno; en este caso, especialmente el gobierno local. En su trabajo, Ostrom ya reconoce la importancia de los gobiernos en legitimar y reducir los costes de la acción colectiva. Sheila Foster (2011), por su parte, señala que, en el ámbito urbano, el rol del gobierno adquiere aún más protagonismo, y ello por diferentes razones. La primera es que es muy difícil encontrar espacios en los que el gobierno local no tenga la propiedad, o al menos un cierto control regulatorio, sobre los recursos. En segundo lugar, porque las comunidades de gestión colectiva a menudo no cumplen las condiciones ideales, por lo que el papel facilitador y legitimador del

4 Siempre teniendo en cuenta que la proximidad residencial no tiene por qué generar vecindad (en el sentido de apoyo mutuo o de comunidad).

5 En palabras de Ostrom: "Because the individuals involved gain a major part of their economic return from the cprs, they are strongly motivated to try to solve common problems to enhance their own productivity over time". 
Estado adquiere todavía más importancia en el funcionamiento óptimo del recurso. La autora realiza el símil de la "tragedia de los comunes" en la ciudad cuando el gobierno local se desresponsabiliza de algunas de sus funciones de regulación y los bienes comunes empiezan a degradarse por la sobreexplotación o el mal uso por parte de los usuarios. Es lo que denomina "decadencia regulatoria"6: "Para decirlo de forma sencilla: la decadencia regulatoria se refiere a un marcado declive en el cumplimiento de las normas o a un aumento de la tolerancia hacia el cumplimiento de las normas por parte de los usuarios del espacio público (...) el concepto de decadencia regulatoria hace referencia simplemente al declive en la gestión o el control de aquellos recursos comunes respecto de cuyo gobierno las autoridades públicas tienen autoridad formal” (p. 67). La autora no entra en las causas de esta decadencia más allá de apuntar una posible insuficiencia financiera o bien un exceso de demanda por parte de la población usuaria. Es, sin duda, una perspectiva muy naif sobre el gobierno local, su capacidad de agencia, sus constreńimientos o los intereses que lo guían en cada momento.

En los casos de gestión colectiva analizados por Foster (huertos urbanos, cuidado de parques públicos, coaliciones para el desarrollo local y patrullas ciudadanas para la seguridad), el rol que otorga al gobierno local es sobre todo el de dar apoyo, por ejemplo reduciendo los costes de involucrarse en experiencias de acción colectiva, desincentivando a los "gorrones", etcétera. Así, clasifica las diferentes experiencias de comunes urbanos según el grado de "apoyo" del gobierno local. Este apoyo vendría determinado sobre todo por las variables endógenas de la comunidad (por ejemplo, si hay lazos fuertes, el apoyo del gobierno local sería menos necesario).

Estos elementos de alguna manera hacen referencia a uno de los principios que garantizan una gobernanza sostenible de los recursos comunes, según la clásica lista de Ostrom. Sin embargo, tal y como destaca, entre otros, De Angelis (2003): "La obra de la historia nos muestra que el reconocimiento de la autodeterminación de la comunidad por parte de autoridades de alto nivel (como el Estado) se gana a menudo a través de la lucha” (p. 220).

\section{La economía política de los comunes}

En la segunda parte de este artículo vamos a movernos a un terreno diferente, retomando aportaciones que, más allá de evaluar si el resultado de la gestión colectiva de recursos comunes es considerado trágico o no, estudian el fenómeno en su relación (antagonista) con el capital, situando esta relación como uno de los campos centrales del conflicto y la resistencia en el capitalismo contemporáneo.

El planteamiento o hipótesis, compartido por autores que podríamos situar dentro de la corriente neomarxista o de un marxismo heterodoxo, es el siguiente:

6 Traducción propia de "regulatory slipperage". La traducción de todos los textos no castellanos es de los autores.

7 Aquí nos referimos al conocido como el problema del gorrón (término que, entre otras acepciones, la Real Academia Española define como "que tiene por hábito comer, vivir, regalarse o divertirse a costa ajena") o "free rider": el hecho de que un individuo pueda usar el bien aun sin haber contribuido a su provisión, debido a la dificultad de exclusión. 
la estrategia de acumulación en el neoliberalismo y la forma rentista que adopta el beneficio bajo la hegemonía del capital financiero, se asemejan bastante al proceso de cercamiento o enclosures ${ }^{8}$ de los campos comunales descrito por Marx en el marco de lo que denomina "acumulación primitiva". (Véase, entre otros, De Angelis, 2003; Federici, 2004; Hardt, 2010; Hardt \& Negri, 2011; Harvey, 2004; Heynen \& Robbins, 2005; Marazzi, 2009; Vercellone, 2009).

En 1990, el mismo año en que Ostrom publicaba su Governing the Commons, el colectivo editorial Midnight Notes acuñó el término "nuevos cercamientos" ("new enclosures") para referirse al proceso de privatización espectacular y sin precedentes que estaba teniendo lugar a lo largo y ancho del planeta desde mediados de 1970 en adelante, como resultado del giro neoliberal global (Midnight Notes Collective, 1990).

En la misma línea, David Harvey (2003) recupera y adapta la noción de "acumulación primitiva" de Marx bajo el epígrafe de "acumulación por desposesión”, con el que describe el nuevo cierre de los campos comunales como fórmula para superar los problemas de sobreacumulación. Refiriéndose a este retorno de la acumulación primitiva, Hardt y Negri (2009) insisten en que esta no puede ser leída simplemente como un proceso de apropiación de recursos físicos, sino que debe ser considerada un proceso de usurpación de "la productividad del trabajo vivo". Žižek (2009), por su parte, define cuatro antagonismos fundamentales que constituyen la principal y actual amenaza a los comunes: la catástrofe ecológica, el funcionamiento de los regímenes de propiedad privada (pone como ejemplo las patentes y la propiedad intelectual), los desarrollos tecnocientíficos (especialmente la biogenética) y la emergencia de nuevas formas de apartheid (muros, vallas y fronteras).

\section{Los "nuevos cercamientos" de la ciudad neoliberal}

A pesar del creciente reconocimiento de la utilidad de las teorías de la acumulación primitiva marxistas para entender la actual fase neoliberal del desarrollo capitalista, diversos autores han destacado su alto nivel de abstracción y poca concreción empírica, la falta de investigación sobre la dinámica particular que la desposesión y los cercamientos asumen en la escala urbana (Hart, 2006; Hodkinson, 2012), así como la relativa ausencia de investigación sobre las prácticas contemporáneas de “comunalización urbana” (Bresnihan \& Byrne, 2015).

Recientemente ha aflorado una nueva línea de producción académica que, entroncada en la teoría urbana crítica, desarrolla una mirada renovada, que vincula las aportaciones de los teóricos marxistas de la acumulación primitiva con los trabajos sobre el neoliberalismo en las ciudades, estudios provenientes de la

Arcaísmo asociado al capítulo fundacional del capitalismo y "desenterrado" recientemente, es el de "enclosure" o cercamiento. El término hace referencia a los protocolos legales que supusieron la expulsión de los campesinos desde los campos comunales y la transformación de estos campos en parcelas de propiedad privada, tal y como sucedió con gran parte de las tierras comunales en la Inglaterra de los siglos xviII y xix. Marx lo describe como uno de los diferentes métodos "sangrientos" que conforman el "pecado original" que permitió la acumulación de capital y trabajo necesaria para la transición al capitalismo, proceso conocido como acumulación primitiva u originaria. 
geografía crítica y la sociología urbana (véase Blomley, 2008; Hodkinson, 2012; Jeffrey, McFarlane \& Vasudevan, 2012; Vasudevan, McFarlane \& Jeffrey, 2008). Estos autores recuperan el concepto de "cercamiento" para referirse al proceso de "neoliberalización" de las ciudades, poniendo así en el centro no solo lo que se entiende coloquialmente por "privatización" -la transferencia de activos públicos al ámbito privado-, sino también una multiplicidad y diversidad de actos, que van desde la privatización/financiarización del espacio urbano y la mercantilización de la vida urbana, a la polución, la exclusión, el desplazamiento o el levantamiento literal de cercas y muros.

Stuart Hodkinson (2012) describe los "cercamientos" urbanos no solo como "una metáfora de la política urbana contemporánea (...) sino [como] el modus operandi del urbanismo neoliberal basado en: a) Identificar nuevas salidas urbanas para reiniciar el ciclo de acumulación; b) Controlar el espacio urbano, ya sea en su valor de uso o en su valor mercantil; c) Impedir cualquier espacio o forma de sociabilidad que ofrezca un medio de reproducción (alejado de la lógica de la producción de las mercancías) y suponga un desafío a las relaciones sociales capitalistas” (p. 505).

Podemos ordenar las diferentes perspectivas en tres grandes bloques: i) aquellas que se centran en los procesos de fragmentación, privatización y securitización de los espacios urbanos; ii) aquellos enfoques que amplían la escala abordando las dinámicas gentrificadoras como procesos de cercamiento de barrios enteros por parte del capital, y iii) aquellos que abordan las "externalidades negativas" de modelos de acumulación basados en un uso intensivo de los activos territoriales (polución, agotamiento de recursos y homogeneización), entendiendo la ciudad como el soporte de otros comunes que sobre ella se asientan.

En primer lugar, observamos cómo la literatura ha utilizado el concepto de "cercamiento" para hacer referencia a la expansión e intensificación de cercas, muros, fronteras o fortificaciones que privatizan y mercantilizan los espacios públicos anteriormente abiertos y accesibles, en favor de determinadas elites urbanas "desplazando" y "excluyendo" a los pobres urbanos de la ciudad. Alex Jeffrey, Colin McFarlane y Alex Vasudevan (2012) analizan esta proliferación de arquitecturas de seguridad como "la forma más rudimentaria y geográficamente evidente de cercamiento". Como ejemplos citan las Zonas Económicas Especiales (ZES) o los barrios o condominios cerrados ("gated communities"), donde las clases más favorecidas por el proceso de globalización se aíslan del resto de la ciudad generando hábitats protegidos. De alguna manera, todos estos procesos pueden recordar los cercamientos y expulsión de los campesinos de los campos comunales en los inicios del capitalismo: vallado, mercantilización y privatización de la tierra, expulsión de las clases populares (en este caso, expulsión desde los centros históricos) para poner la tierra al servicio de usos más lucrativos, y la transformación de los derechos comunes-como puede ser el derecho al espacio público- en derechos privados.

Otro ejemplo, citado en la literatura como "cercamiento urbano" y que mantiene fuertes paralelismos con las dinámicas que dieron pie a la formación histórica de la clase obrera, es el de la gentrificación (Atkinson, 2000; Smith, 2002), concepto que sintetiza una serie de procesos encaminados a la reconquista de espacios centrales a través del encarecimiento de la vivienda y los servicios del barrio, y que generan, 
entre otros elementos, el desplazamiento (directo e indirecto) de la población anterior y una erosión de las redes sociales de las clases populares (Blomley, 2008). En este sentido, el proceso de cercamiento no solo hace referencia a los intentos corporativos de controlar, privatizar y comercializar el espacio público, sino que se amplía a los recursos generados por la vida urbana: la cultura, el patrimonio, el capital simbólico, los estilos de vida, etcétera.

Harvey (2012) da un buen ejemplo de la inversión de "la tragedia de Hardin" cuando describe la gentrificación como ejemplo paradigmático de cómo, por excesiva deferencia a la propiedad privada y al interés individual, se produce una degradación y mala gestión de un recurso común: "Cuanto mejores son las cualidades comunes que un grupo social crea, es más probable que ellas sean apropiadas por intereses privados maximizadores de beneficios (...) y una vez que el mercado ha hecho su trabajo destructivo, no solo los residentes originales han sido desposeídos de ese común que habían creado (a menudo forzados a abandonar el barrio por el aumento de alquileres), sino que el común en sí mismo llega a estar tan devaluado que se hace irreconocible" (p. 78).

Tal y como lo reconocen, entre otros, Gago y Mezzadra (2015), haciendo referencia a la literatura que aborda la política de desarrollo "neoextractivista" en países de Latinoamérica, la extracción no solo sucede en reservas naturales o áreas indígenas rurales, sino que las ciudades también son objeto de operaciones de extracción, llevadas a cabo mediante la desposesión social de bienes comunes urbanos a partir de procesos como la gentrificación. No solo la comunidad se ve desposeída del común que ha producido (en este caso, el particular ecosistema urbano, el ambiente, la diversidad, etcétera) debido al aumento de los precios del suelo y de las viviendas, sino que los efectos trivializantes y homogeneizantes del mercado suponen un agotamiento de este particular ecosistema urbano.

Junto con lo anterior, el término "cercamiento" es utilizado por ciertos autores para analizar los proyectos de demolición y reconstrucción de zonas urbanas degradadas, como favelas o barrios de chabolas, por considerarlo un término analíticamente más robusto que "gentrificación", puesto que permite vincular (tanto conceptual como políticamente) las luchas contra los desplazamientos que se dan en el norte y en el sur global (Ghertner, 2014).

Por último, junto al proceso de vallado (literal o metafórico) -esto es, el desplazamiento y la absorción de la riqueza colectiva en beneficio de determinados sectores productivos y especulativos-, el proceso de cercamiento ha sido utilizado como marco para abordar la degradación de recursos comunes y la contaminación en entornos compartidos, lo que los economistas denominan como las externalidades negativas del mercado: "Lo que el mercado considera externalidades incidentales, la narrativa de los bienes comunes lo considera como una agresión a la comunidad" (Bollier, 2011, p. 49).

Utilizando el caso español durante los años que duró el último boom inmobiliario (1993-2007) como ejemplo ilustrativo, los estudios del Observatorio convirtió en la tragedia de su desaparición. 
Metropolitano $(2007 ; 2011 ; 2013)$ describen de forma detallada cómo un modelo basado en la propiedad inmobiliaria, en los ciclos patrimoniales y en la masiva construcción de infraestructuras, se desarrolla necesariamente sobre la materialidad del territorio a partir de una sustracción constante y un uso intensivo de los activos naturales comunes. Según los autores, esta "colonización del territorio" supone que recursos que pertenecen a toda la colectividad, como el litoral, el aire, el suelo urbano o el agua, entre otros, experimenten procesos de sobreexplotación y contaminación que ponen en peligro su sostenibilidad: "Muchos recursos comunes están siendo privatizados y mercantilizados porque las corporaciones ven forraje barato o gratuito para la máquina voraz del mercado (...) al mismo tiempo, representan un barato y conveniente basurero -un lugar para deshacerse de todas las externalidades desagradables que las empresas no quieren a internalizar en sus estructuras de costos" (Observatorio Metropolitano, 2013, p. 28).

En resumen, mientras para visiones de corte neoclásico, el cercamiento es sin duda un sinónimo de aumento de la productividad o rentabilidad ante la supuesta inestabilidad inherente a los régimenes de propiedad colectiva (Hardin, 1968; Lee \& Webster, 2006), desde estas perspectivas representa una lógica que subyace a diversas dinámicas y cambios sociales no deseados que se producen en las metrópolis contemporáneas, impulsados por la lógica expansiva del capitalismo de libre mercado (véase Blomley, 2008; Hodkinson, 2012; Jeffrey et al., 2012; Vasudevan et al., 2008).

Es precisamente en los intersticios del proceso de privatización de los comunes urbanos que deben situarse procesos actuales clave de transformación políticoeconómica urbana, de neoliberalización y financiarización. Esto se ha vuelto más acuciante a la luz de los procesos extraordinarios de desposesión que se están desarrollando a raíz de la fusión de la crisis financiera con el conjunto de medidas de austeridad y rectitud fiscal (Midnight Notes Collective and Friends, 2009). Y aunque la fuerza evocadora del término "cercamiento" ha proporcionado una potente metáfora sobre la expansión del poder empresarial en la ciudad, esta concepción tan laxa y difusa ${ }^{10}$ ha generado debates sobre su validez analítica y explicativa (Serlin \& Chazkel, 2011; De Angelis, 2003; Harvey, 2004).

\section{Reclamar el derecho a la ciudad a través de afirmar lo común}

Hasta ahora hemos contemplado las ciudades como lugares privilegiados de la "acumulación por desposesión” (Harvey, 2004) bajo el neoliberalismo. Sin embargo, también son y han sido espacios de fricciones y resistencia, altamente disputados, escenarios de luchas de carácter cotidiano y de otras explícitamente políticas, que han sido identificadas y leídas, bajo el prisma de los comunes, como "el afuera" (the outside) de los cercamientos (De Angelis, 2004).

10 Tal y como nos recuerda De Angelis (2003), la literatura actual no ofrece mucho en términos de clasificación sistemática de los diferentes mecanismos a través de los cuales operan los cercamientos y, menos aún, en el caso específico de los cercamientos urbanos. A su vez, tal y como nos recuerda David Harvey al referirse a las gated communities, la dinámica comunes-cercamiento no puede ser leída de forma simplista y lineal, sino que debe tenerse en cuenta el contexto, ya que lo que se considera un común en un contexto puede llegar a ser una propiedad privada en otro, y viceversa. 
Desde que el colectivo Midnight Notes (1990) o autores como Naomi Klein, en el influyente "Reclaiming the commons" (2001), describieron el ciclo global de luchas iniciado a principios de los noventa (el denominado movimiento anti o alterglobalización) como una batalla para "recuperar los bienes comunes" y "reclamar el control de aquello que estaba siendo privatizado", se inició un debate en los estudios urbanos críticos que da cuenta de la creciente importancia otorgada a los comunes por parte de los movimientos políticos. (Véase Caffentzis, 2010; De Angelis, 2003; De Peuter \& Dyer-Witheford, 2010; Fattori, 2013; Hardt \& Negri, 2011; McCarthy, 2005; o Stravides, 2011, para una revisión).

David Harvey argumenta que la acumulación por desposesión ha dado lugar a una gran variedad de luchas que muestran una serie de características nuevas, al no tener lugar en la fábrica sino en la ciudad y no estar lideradas por la clase trabajadora sino por un amplio espectro de movimientos sociales y de la sociedad civil. El autor retoma una idea que ya sugirió Henri Lefebvre en su momento al abordar las raíces urbanas del movimiento de mayo del 68 en París. El capital ha salido de los muros de la fábrica y el territorio ha devenido "la fábrica social". Este hecho otorga un papel cada vez más primordial a las luchas urbanas como fuente de resistencia al capitalismo, desplazando a las luchas proletarias. Hardt y Negri (2011) van en una línea parecida cuando señalan que "la metrópoli es a la multitud lo que la fábrica era a la clase obrera” (p. 250). De este modo, y simplificando, mientras las luchas "obreras" están orientadas a conseguir mejoras en las condiciones laborales, las movilizaciones contra la desposesión están orientadas a reclamar y recuperar los bienes comunes como condición que posibilita la vida en la gran fábrica social que es la ciudad.

En su último libro, Ciudades rebeldes (2012), Harvey analiza la importancia de la dimensión urbana en el ciclo de luchas que comenzó en 2010-2011 en Tahir, en la plaza Syntagma, en el parque Gezi, en las acampadas de los indignados del 15M, y señala cómo los comunes urbanos -en particular, las calles, plazas y parques de las ciudades- se convierten en el principal lugar de resistencia y reivindicación. A la vez, destaca los actos de ocupación y reapropiación del espacio público y cómo las acciones colectivas generadas en el crisol de la vida urbana a las que hace mención, pueden ser leídas como un acto de "comunalización": transforman los lugares (la plaza Syntagma en Atenas, la plaza Tahrir en El Cairo, el parque Gezi en Estambul, el parque Zuccotti en Nueva York) en un común urbano, reformulando su gestión a partir de bases colectivas y desafiando la forma en que el espacio público urbano es controlado y administrado: "poner los cuerpos humanos en este lugar [plazas, parques y calles], convierte el espacio público en un común político, un lugar para el debate y la discusión abierta sobre las maniobras del poder (...). Las plazas Syntagma en Atenas, Tahrir en El Cairo y de Catalunya en Barcelona eran espacios públicos que se convirtieron en un bien común urbano cuando la gente se reunió allí para expresar sus opiniones políticas y proclamar sus reivindicaciones" (p. 161).

Inspirados en esta noción de Harvey de reapropiación de la condición urbana como un común, y a partir de un estudio etnográfico sobre los "indignados del 15M” en España, Estalella y Corsín (2013) describen cómo las plazas -campamentos urbanos temporales que recrean "ciudades en miniatura" (p. 151)-, 
hostigadas por la privatización de su uso y por el autoritarismo y las prohibiciones, emergieron como un común urbano a partir de la organización asamblearia en el espacio público, distribuida y rizomática en la red, conformando un nuevo modo de "hacer o estar-en-común". Salingaros (2010) ha descrito esta forma social creada en las propias prácticas de hacer común bajo el término "urbanismo P2P" o Persona-aPersona (peer-to-peer, por sus siglas en inglés), donde aplica las ideas y las prácticas de los movimientos del software libre a diferentes comunidades en la ciudad que generan formas alternativas de acceder, producir y valorar el espacio urbano a partir de lógicas de código abierto y cooperación social "para definir un espacio urbano para su uso por la gente" (Salingaros, 2010, p. 26). Stravides (2011) define estas "comunidades en movimiento" o "comunidades-en-el-hacer" como "comunidades, creadas en una sociedad en movimiento a través de las actividades catalíticas de los movimientos sociales urbanos" (p. 3). Estas organizaciones sociales están lejos de la idea de comunidad homogénea y de corte esencialista de las comunidades rurales o de los lazos sociales de la familia extensa, ya que, según el autor, se fundamentan en el hacer común; es decir, aparecen a raíz de la movilización y la acción colectiva y se constituyen en la propia acción de gestionar y democráticamente gobernar recursos comunes: "La comunidad se desarrolla a través del commoning, a través de actos y formas de organización orientados hacia la producción del común” (p. 5).

Aquí es importante destacar lo siguiente: es precisamente esta práctica social de comunalización la que convierte bienes, servicios y espacios públicos, desde una plaza a la educación pública, en recursos comunes. Esto va en la línea con lo que plantea Peter Linebaugh (2010) cuando convierte el nombre (bienes comunes) en verbo (“commoning") para destacar cómo lo común, más allá de un objeto o recurso físico (RC), no puede existir sin el acto de comunalización, es decir, sin la acción de una determinada comunidad que decide defender y administrar un recurso de manera colectiva. En este sentido, los comunes no solo presuponen una comunidad, sino que son producidos en la lucha.

Un buen ejemplo de esto puede ser extraído de los trabajos del geógrafo Nicholas Blomley (2007; 2008). En uno de sus artículos ("Enclosure, Common Right and the Property of the Poor", 2008), el autor destaca la continua apelación que hace una comunidad de vecinos a la propiedad colectiva de su barrio ("Woodwards Belongs To Us") como forma de enfrentarse a un proceso de regeneración "agresivo" y poner límites al derecho exclusivo de los promotores inmobiliarios y a su capacidad para desplazar a las poblaciones pobres. El desafío a la capacidad del Estado y del capital para sostener su duopolio en las tareas de provisión de servicios o planificación urbana es lo que permite al autor leer la movilización como un común urbano. A su vez, la movilización ha sido particularmente activa contra la apropiación de las ciudades por los intereses privados a partir de la interpelación y defensa de lo urbano como un común, afirmando principios de acceso, participación y sostenibilidad, los que se encuentran en el corazón de la lucha por los comunes. Aquí, lo común, lejos de considerarse como experiencias aisladas de pequeñas comunidades que gestionan recursos, emerge y se presenta como una idea filosófico-política que se articula mediante el lenguaje de los derechos: derecho a la propiedad colectiva (de la ciudad) en contraposición a la ciudad como agregación de derechos de propiedad 
privada $^{11}$ ). Esta concepción de los derechos que subyace en la práctica de hacer y reclamar el común, permite conectar con la literatura que aborda las luchas por "le droit à la ville", concepto acuñado por Lefebvre para abordar las movilizaciones del mayo francés del 62 (Lefebvre, 1968). En este sentido, destaca la introducción de elementos clave ausentes en muchas investigaciones neoinstitucionalistas, como, por ejemplo, la centralidad de cuestiones éticas o el papel medular de la contestación política: "Los comunes no son tanto algo encontrado, como producido" (Blomley, 2008, p. 320).

Por otro lado, tenemos a los autores de lo que se conoce como el ámbito de la autonomía marxista italiana o del posoperaísmo, como Hardt y Negri (2011). Estos autores sitúan la cuestión de "lo común" o "la producción de lo común" como un elemento central en el "capitalismo cognitivo" y en la forma en que este asume una doble condición antagónica: es tanto el plano de la autonomía como el de la captura capitalista (Hardt \& Negri, 2009; Pasquinelli, 2008; Roggero, 2010; Vercellone, 2009). Por un lado, el territorio mismo, la metrópoli y no ya la fábrica, es considerado la base de la innovación social, de la "producción biopolítica", al ser la fuente y el espacio de lo común "de la gente viviendo junta, compartiendo recursos, comunicando, intercambiando bienes e ideas" (Hardt \& Negri, 2011, p. 133). Esta riqueza producida socialmente se ve subsumida por el capital, principalmente a través de diferentes mecanismos financieros y del control y la apropiación de las rentas urbanas, beneficiando a ciertos sectores dominantes dentro de la economía de la ciudad (turismo, inmobiliario, financiero).

Las visiones de estos autores amplían las definiciones de los comunes presentes en los estudios neoinstitucionalistas (que se refieren siempre a "pequeñas comunidades de usuarios"), hasta abarcar todos aquellos recursos o bienes, espacios y formas de sociabilidad en la ciudad que son de carácter colectivo ("son de todos"), tanto en términos de propiedad ("la riqueza heredada") como de producción ("los productos y resultados del trabajo individual y colectivo"), y que forman la base para promover la vida productiva de la metrópoli y satisfacer las necesidades básicas. Por tanto, más allá de reivindicaciones específicas para gestionar algunos recursos comunes concretos de forma colectiva, según estos autores, una "democracia de lo común" se basa en generar instituciones que permitan gestionar democráticamente la producción o riqueza colectiva. Las luchas por "el común", por tanto, apuntan hacia una crítica "general" a las dicotomías público-privado, es decir, al neoliberalismo y a sus privatizaciones, así como al papel del sector público y su falta de democracia y transparencia.

En resumen, observamos cómo el concepto de "bienes comunes" es cada vez más móvil, ampliándose más allá de su significado original relativo a un recurso físico, para encontrar aplicación como un instrumento de análisis de los conflictos urbanos y de la contestación política como resultado de la extensión del urbanismo neoliberal. Sea expresado en términos ideológicos o suponga una respuesta pragmática a necesidades materiales (Bresnihan \& Byrne, 2015), sea que se refiera a procesos

$1 1 \longdiv { \text { La propiedad privada como un atributo absoluto del propietario (ya sea individual o institucional) } }$ que le confiere (a este) la capacidad de decidir sobre un objeto, o en este caso (los derechos exclusivos sobre) el uso y explotación de la ciudad. 
de autogestión, como movimientos de defensa y reapropiación de derechos (Observatorio Metropolitano, 2013), esta forma social creada en las propias prácticas de hacer común articula una crítica al urbanismo neoliberal, al desafiar la lógica hegemónica de la propiedad privada así como el funcionamiento y el papel de las instituciones públicas. Tal y como nos recuerda Chatterton, "el común urbano y las prácticas y las relaciones sociales que lo respaldan, se han convertido en la más pura expresión del tipo de política necesaria para una mayor justicia en la ciudad (...). Al mismo tiempo un acto defensivo y un acto productivo contra los cercamientos y las opresiones" (Chatterton, 2010, p. 627).

\section{Conclusiones}

Aunque términos como "cercamiento" (enclosures) o bienes comunes (commons) han sido asociados normalmente a épocas pasadas, estas nociones "extrańas y arcaicas" han experimentado un renovado interés en los últimos tiempos por parte de movimientos sociales y diferentes académicos de variadas disciplinas.

En este artículo hemos explorado dos aproximaciones a la literatura sobre los comunes y sus derivadas en los estudios urbanos (cuadro 1). En la primera, hemos aplicado la perspectiva sobre la gestión colectiva de bienes comunes en la ciudad, muy influenciada por los trabajos de Elinor Ostrom sobre las condiciones para desarrollar una gestión colectiva eficaz de determinados recursos naturales. En este sentido, hemos destacado la especificidad urbana a la hora de aplicar esta perspectiva en la ciudad: i) la naturaleza y diversidad propia de los recursos urbanos, que son socialmente construidos; ii) el tipo de comunidades que se generan en el ámbito urbano, mucho más abiertas y fluctuantes, y su relación con el recurso (menos dependiente); y, finalmente, iii) el papel del gobierno local en la regulación de estos recursos.

Estas apreciaciones no invalidan de ningún modo la posibilidad de que en el ámbito urbano existan comunidades que autogestionan recursos urbanos de una forma sostenible, aumentando las externalidades positivas en términos de creación de capital social local y de revitalización de la ciudad en su conjunto.

Epistemológicamente, mucho trabajo teórico sobre la gobernanza de los "common pool resources" toma en cuenta que el mundo está poblado por individuos racionales maximizadores de utilidad y, desde ahí, se preguntan bajo qué condiciones es posible y socialmente eficiente el funcionamiento de un régimen de propiedad común (véase, por ejemplo, Ostrom, 1990). Frente a esta perspectiva basada en el individualismo metodológico, las corrientes neomarxistas recuperan la matriz histórica de los bienes comunes, contextualizando el análisis en el marco de las relaciones de poder capitalista. En este sentido, los comunes no son solo una rica variedad más o menos exitosa de modelos de gestión de recursos, sino que lo común aparece como un concepto central para la comprensión de las bases de la producción de la riqueza social y de los modos de acumulación en el capitalismo contemporáneo, siendo, por tanto, fundamental abordar el proceso de "cercamiento" que se produce en la ciudad. 
CUADro I | Perspectivas neoinstitucionalistas y neomarxistas respecto de los comunes urbanos

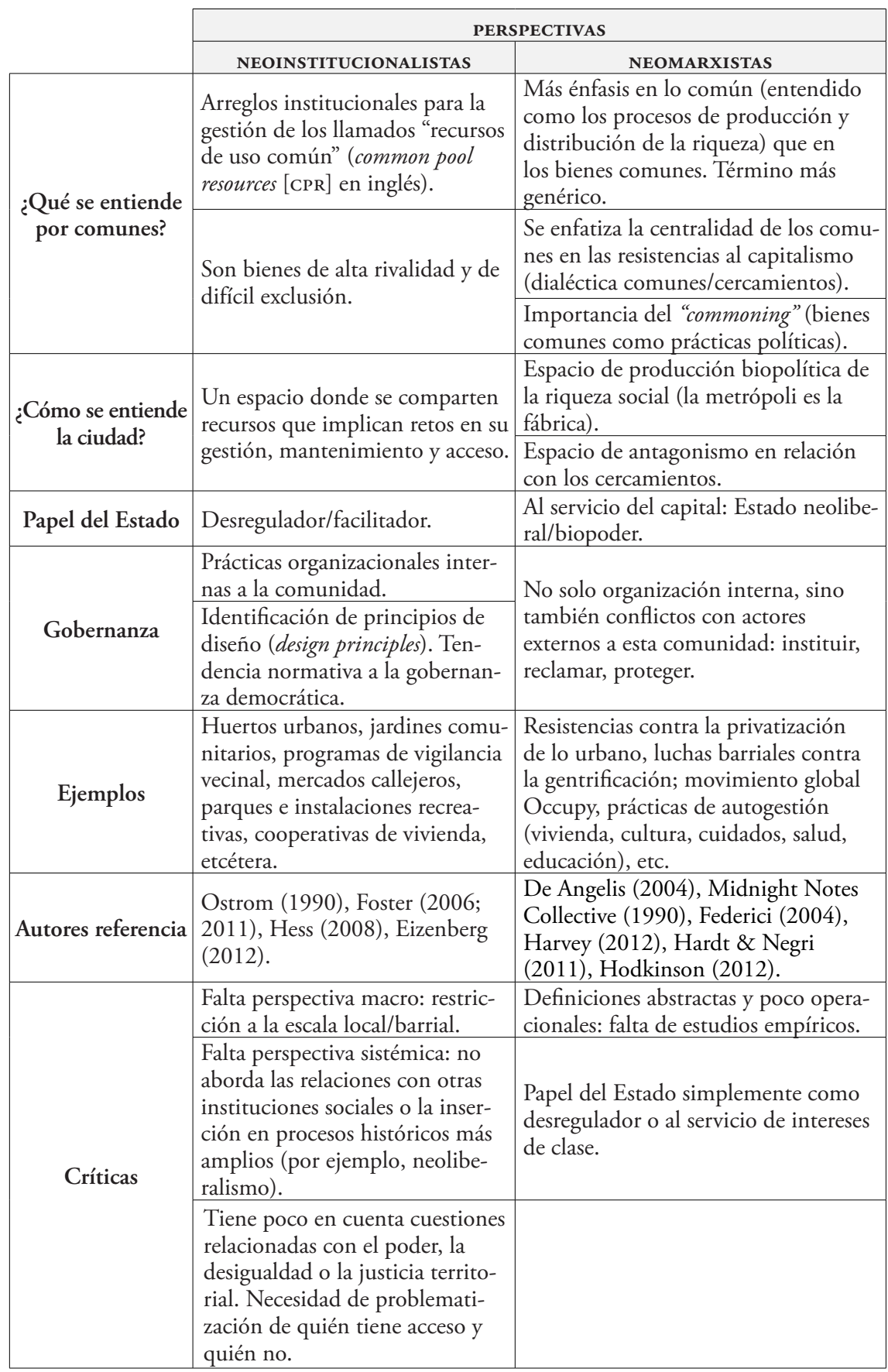

FUENTE ELABORACIÓN PROPIA 
Lo interesante de esta perspectiva a la hora de analizar los comunes urbanos es entender el contexto estructural en el cual se sitúan las tensiones respecto al control de los recursos urbanos. Así, además de entender los comunes urbanos como un recurso, una comunidad y un conjunto de normas que regulan su gestión colectiva, como lo hacen las perspectivas más institucionalistas, se introduce el tema del conflicto, la politización e incluso la reclamación de derechos que van más allá de los propios recursos en sí.

Esta lectura más compleja de los comunes urbanos nos permite, a su vez, introducir otros factores para intentar explicar, entre otras cuestiones, los efectos negativos que algunos comunes pueden producir más allá de sus fronteras o la sostenibilidad de las prácticas en el ámbito urbano. Si observamos únicamente la comunidad que conforma una comunidad residencial cerrada (gated community), sin un abordaje complejo, no podremos explicar, por ejemplo, el vínculo entre el crecimiento de este tipo de productos inmobiliarios y la extinción de otros recursos comunes ligados históricamente a la ciudad, como puede ser la esfera pública; ni tampoco evaluar sus efectos sociales negativos, como el fomento de la división y de la segregación social, o la forma en que favorecen la mercantilización de recursos como la seguridad (Kohn, 2004). Por otro lado, como hemos apuntado, la menor dependencia directa para la subsistencia de los comuneros urbanos respecto de los recursos que gestionan, rebaja los incentivos para mantener la acción colectiva de forma continuada, por lo que son necesarios otros incentivos, tanto individuales como colectivos, para sostener su gestión colectiva.

En este sentido apuntamos dos aspectos que deben ser incorporados para el estudio de la sostenibilidad de los comunes urbanos. En primer lugar, la politización de los participantes en la defensa de un bien común que se considera amenazado por las lógicas privatizadoras, actúa como un importante elemento motivacional para sostener las prácticas comunales (aunque también puede ser un elemento desmotivador cuando las perspectivas de éxito son escasas). En segundo lugar, las perspectivas institucionalistas nos aportan mayor complejidad al estudio del gobierno local, entendido no solamente como un instrumento per se facilitador de los intereses de la burguesía local, estatal o internacional, sino también como posible facilitador de las prácticas cooperativas. Aunque desde las perspectivas neomarxistas la crítica de la forma del Estado moderno es un requisito fundamental para cualquier proyecto político radical democrático, autores como Harvey han intentado complejizar el análisis de la relación entre los comunes y el Estado, al plantear que, por encima de una cierta escala, se requiere algún tipo de estructura de Estado, así como reivindicar su papel como redistribuidor de la riqueza. Aunque las prácticas de autogestión pueden favorecer modos más democráticos de participación a nivel local, el papel del Estado no puede ser ignorado. En esta línea se hace necesario explorar las alianzas comunales-estatales que se puedan desarrollar en cada contexto y ámbito en particular, ampliando lo que se considera como público más allá de lo estrictamente estatal.

El diálogo entre ambas trayectorias reconstruye los bienes comunes urbanos como espacios políticos que aglutinan tanto los procesos de cercamiento y las prácticas de resistencia, como las diferentes experiencias de gestión colectiva que se producen alrededor de ellos. No podemos reducir los comunes urbanos a las experiencias 
de gestión colectiva en la ciudad ni a los movimientos urbanos de defensa de lo público. El estudio de los comunes urbanos bajo la perspectiva compleja que hemos desarrollado en este artículo requiere de una nueva agenda política y de investigación para el estudio de aquellas prácticas sociales y comunitarias que desbordan lo estatal y se rebelan contra las formas de mercantilización y privatización de la vida urbana, para hacer efectivo el derecho colectivo a la ciudad.

\section{Referencias bibliográficas}

Atkinson, R. (2000). The hidden costs of gentrification: Displacement in central London. Journal of Housing and the Built Environment, 15(4), 307-326. doi: 10.1023/A:1010128901782

Bailey, S. \& Mattei, U. (2012). Social movements as constituent power: The Italian struggle for the commons. Indiana Journal of Global Legal Studies, 20(2), 965. En http://bit. ly/1OA8upd

Bakker, K. (2007). The "commons" versus the "commodity": Alter-globalization, antiprivatization and the human right to water in the global South. Antipode, 39(3), 430455. doi: $10.1111 / \mathrm{j} .1467-8330.2007 .00534 . x$

Bennett, T., Holloway, K. \& Farrington, D. P. (2006). Does neighborhood watch reduce crime? A systematic review and meta-analysis. Journal of Experimental Criminology, 2(4), pp. 437-458. doi: 10.1007/s11292-006-9018-5

Blomkvist, P. \& Larsson, J. (2013). An analytical framework for common-pool resource-large technical system (CPR-LTS) constellations. International Journal of the Commons, 7(1), 113-139. En http://www.thecommonsjournal.org/index.php/ijc/article/view/353/317

Blomley, N. (2007). Making private property: Enclosure, common right and the work of hedges. Rural History, 18(1), 1. doi: http://dx.doi.org/10.1017/S0956793306001993

Blomley, N. (2008). Enclosure, common right and the property of the poor. Social \& Legal Studies, 17(3), 311-331. doi: 10.1177/0964663908093966

Bollier, D. (2011) The healing logic of the commons. Kosmos (Fall/Winter 2011), 45-50. En http://www.kosmosjournal.org/wp-content/article-pdfs/the-healing-logic-of-thecommons.pdf

Bravo, G. \& De Moor, T. (2008). The commons in Europe: From past to future. International Journal of the Commons, 2(2), 155-161. En http://www.thecommonsjournal.org/ index.php/ijc/article/view/98

Bresnihan, P. \& Byrne, M. (2015). Escape into the city: Everyday practices of commoning and the production of urban space in Dublin. Antipode, 47(1), 36-54. doi: 10.1111/ anti. 12105

Byrne, J., Martínez, C. \& Ruggero, C. (2009). Relocating energy in the social commons. Ideas for a sustainable energy utility. Bulletin of Science, Technology \& Society, 29(2), 81-94. En http://www.seu-de.org/Papers/2009_pe_BSTS_relocating_emergy_social_ commons_Byrne_Martinez_Ruggero.pdf

Caffentzis, G. (2010). The future of "The Commons": Neoliberalism's "Plan B" or the original disaccumulation of capital? New Formations, 69(1), 23-41. http://dx.doi.org/10.3898/ NEWF.69.01.2010 
Castells, M. (1977). Movimientos sociales urbanos. Madrid: Siglo XxI.

Cavé, J. (2013). Urban solid waste in southern countries: From a blurred object to common pool resources. En ESEE 2013. Ecological Economics and Institutional Dynamics. 10th International Conference of the European Society for Ecological Economics. Final program (p. 436). Reims-Bruselas-Lille, junio 17-21, 2012. En http://esee2013. sciencesconf.org/

Chatterton, P. (2010). Seeking the urban common: Furthering the debate on spatial justice. City: Analysis of urban trends, culture, theory, policy, action, 14(6), 625-628. doi: $10.1080 / 13604813.2010 .525304$

Colding, J. (2011). Creating incentives for increased public engagement in ecosystem management through urban commons. En E. Boyd \& C. Folke (Eds.), Adapting institutions: Governance, complexity and social-ecological resilience (pp. 101-124). Cambridge, RU: Cambridge University Press.

Colding, J. \& Barthel, S. (2013). The potential of "Urban Green Commons" in the resilience building of cities. Ecological Economics, (86), 156-166. http://dx.doi.org/10.1016/j. ecolecon.2012.10.016

Cooper, D. (2006). "Sometimes a community and sometimes a battlefield": From the comedic public sphere to the commons of speakers" corner. Environment and Planning D: Society and Space, 24(5), 753-775. doi: 10.1068/d1004

Corsín Jiménez, A. \& Estalella, A. (2014). Assembling neighbors: The city as hardware, method, and "a very messy kind of archive". Common Knowledge, 20(1), 150-171. doi: 10.1215/0961754X-2374808.

De Angelis, M. (2003). Reflections on alternatives, commons and communities. The Commoner, 6, 1-14. En http://www.citeulike.org/user/rparlin/article/11897416

De Angelis, M. (2004). Separating the doing and the deed: Capital and the continuous character of enclosures. Historical Materialism, 12(2), 57-87. doi: 10.1163/1569206041551609

De Peuter, G. \& Dyer-Witheford, N. (2010). Commons and cooperatives. Affinities: A journal of radical theory, culture, and action, 4(1), 30-56. En http://journals.sfu.ca/affinities/ index.php/affinities/article/view/45

Eizenberg, E. (2012). Actually existing commons: Three moments of space of community gardens in New York City. Antipode, 44(3), 764-782. doi: 10.1111/j.14678330.2011.00892.x

Estalella, A. \& Corsín, A. (2013). Asambleas populares: el ritmo urbano de una política de la experimentación. En M. Cruells \& P. Ibarra (Eds.), La democracia del futuro: del $15 M$ a la emergencia de una sociedad civil viva (pp. 61-80). Madrid: Icaria. [Borrador publicado en http://digital.csic.es/bitstream/10261/85040/1/asambleas\%20 ritmo\%20urbano\%202013.pdf].

Fattori, T. (2013). From the water commons movement to the commonification of the public realm. South Atlantic Quarterly, 112(2), 377-387. doi: 10.1215/00382876-2020253

Federici, S. (2004). Calibán y la bruja: Mujeres, cuerpo y acumulación originaria. Madrid: Traficantes de Sueños.

Federici, S. (2011). Feminism and the politics of the commons. The Commoner [web journal], 24. En http://www.commoner.org.uk/?p=113 
Fernández, M. \& Burch, W. (2003). Cultivating community, food, and empowerment: Urban gardens in New York City. Project course paper. Fall 2003. New Haven, CT: Hixon Center for Urban Ecology, Yale University. En http://hixon.yale.edu/sites/default/files/ files/fellows/paper/fernandez_margarita_2003_report.pdf

Foster, S. R. (2006). City as an ecological space: Social capital and urban land use. Notre Dame Law Review, 82(2), 527-582. En http://papers.ssrn.com/sol3/papers.cfm?abstract_ $\mathrm{id}=899617$

Foster, S. R. (2011). Collective action and the urban commons. Notre Dame Law Review, 87(1), 57-133. En http://papers.ssrn.com/sol3/papers.cfm?abstract_id=1791767

Frischmann, B. M. (2005). Infrastructure commons. Law Review of Michigan State University, 121(1), 121-136.

Frischmann, B. M. (2006). An economic theory of infrastructure and commons management. American Law \& Economics Association Annual Meetings. Paper 18. The Berkeley Electronic Press. En http://law.bepress.com/alea/16th/bazaar/art18 [Originalmente en Minnesota Law Review, 89, 917-1030, abril 2005, http://papers.ssrn.com/sol3/papers. cfm?abstract_id=588424].

Gago, V. \& Mezzadra, S. (2015). Para una crítica de las operaciones extractivas del capital. Patrón de acumulación y luchas sociales en el tiempo de la financiarización. Nueva sociedad, (255), 38-52. [Una versión más amplia de este artículo en Euronmade, posted en Dossier América Latina, Español, en http://www.euronomade.info/?p=4390].

Ghertner, D. A. (2014). India”s urban revolution: geographies of displacement beyond gentrification. Environment and Planning A, 46(7), 1554-1571. En http://www. envplan.com/abstract.cgi?id=a46288

Gutiérrez Aguilar, R. (2008). Los ritmos del Pachakuti. Movilización y levantamiento popularindigena en Bolivia (2000-2005). Buenos Aires: Tinta Limón. En la-periferica.com.ar/ descargar.php?libro=978-987-23140-9-5.pdf

Hardin, G. (1968). The tragedy of the commons. Science 162(3859), 1243-1248. doi: 10.1126/ science.162.3859.1243

Hardt, M. (2010). The common in communism. Rethinking Marxism, 22(3), 346-356. doi: 10.1080/08935696.2010.490365

Hardt, M. \& Negri, A. (2011). Commonwealth: El proyecto de una revolución del común. Colección Cuestiones de Antagonismo, 64. Madrid: Akal.

Hart, G. (2006). Denaturalizing dispossession: Critical ethnography in the age of resurgent imperialism. Antipode, 38(5), 977-1004. doi: 10.1111/j.1467-8330.2006.00489.x

Harvey, D. (2004). El nuevo imperialismo. Colección Cuestiones de Antagonismo, 26. Madrid: Akal.

Harvey, D. (2012). Rebel cities: From the right to the city to the urban revolution. Londres: Verso.

Hernández, I. L. \& López, E. R. (2010). Fin de ciclo: financiarización, territorio y sociedad de propietarios en la onda larga del capitalismo hispano (1959-2010). Madrid: Traficantes de Sueños.

Hess, C. (2008). Mapping the new commons. Trabajo presentado en The Twelfth Biennial Conference of the International Association for the Study of Commons, Cheltenham, Inglaterra, 14-18 de julio 2008. En http://surface.syr.edu/cgi/viewcontent. cgi?article $=1023 \&$ context $=$ sul 
Heynen, N. \& Robbins, P. (2005). The neoliberalization of nature: Governance, privatization, enclosure and valuation. Capitalism Nature Socialism, 16(1), 5-8.

Hodkinson, S. (2012). The new urban enclosures. City, 16(5), 500-518. doi: 10.1080/13604813.2012.709403 Hodkinson, S. \& Chatterton, P. (2006). Autonomy in the city? Reflections on the social centres movement in the UK. City, 10(3), 305-315. doi: 10.1080/13604810600982222

Jeffrey, A., McFarlane, C. \& Vasudevan, A. (2012). Rethinking enclosure: Space, subjectivity and the commons. Antipode, 44(4), 1247-1267. doi: 10.1111/j.1467-8330.2011.00954.x

Kassa, D. G. (2008). Tragedy of the "urban commons"? A case study of two public places in Addis Ababa. Trabajo presentado en The Twelfth Biennial Conference of the International Association for the Study of Commons, Cheltenham, Inglaterra, 14-18 de julio 2008. En http://dlc.dlib.indiana.edu/dlc/handle/10535/792

Klein, N. (2001). Reclaiming the commons. New Left Review, 9 (mayo-junio), 81-89. En http://newleftreview.org/II/9/naomi-klein-reclaiming-the-commons

Kohn, M. (2004). Brave new neighborhoods: The privatization of public space. Nueva York/ Londres: Routledge.

Lambing, J. (2012). Electricity commons - Toward a new industrial society. En D. Bollier \& S. Helfrich, The wealth of the commons: A world beyond market \& state. Amherst-Florence, MA: Levellers Press.

Le Goix, R. \& Webster, C. (2006). Gated communities, sustainable cities and a tragedy of the urban commons. Critical Planning, 13 (Summer 2006), 41-64. En https://hal. archives-ouvertes.fr/halshs-00110046/document

Lee, S. \& Webster, C. (2006). Enclosure of the urban commons. GeoJournal, 66(1-2), 27-42. doi: 10.1007/s10708-006-9014-3.

Lefebvre, H. (1968). Le droit à la ville. París: Anthropos.

Linebaugh, P. (2010). Enclosures from the bottom up. Radical History Review, 2010(108), 11 27. doi: 10.1215/01636545-2010-007

Low, S. \& Smith, N. (Eds.). (2006). The politics of public space. Nueva York/Londres: Routledge. Versión digitlizada en https://chisineu.files.wordpress.com/2012/08/biblioteca_low_ smith_politics_ps.pdf

Marazzi, C. (2009). La violencia del capitalismo financiero. En S. Mezzadra (Comp.), La gran crisis de la economía global: mercados financieros, luchas sociales y nuevos escenarios políticos (pp. 21-62). Madrid: Traficantes de Sueños.

Matisoff, D. \& Noonan, D. (2012). Managing contested greenspace: Neighborhood commons and the rise of dog parks. International Journal of the Commons, 6(1), 28-51. En http:// www.thecommonsjournal.org/index.php/ijc/article/view/299

McCarthy, J. (2005). Commons as counterhegemonic projects. Capitalism Nature Socialism, 16(1), 9-24. doi: 10.1080/1045575052000335348

McShane, I. (2010). Trojan horse or adaptive institutions? Some reflections on urban commons in Australia. Urban Policy and Research, 28(1), 101-116. doi: $10.1080 / 08111140903487711$

Midnight Notes Collective. (1990). Introduction to the new enclosures. Midnight Notes, 10, 1-9. En http://www.midnightnotes.org/newenclos.html

Midnight Notes Collective and Friends. (2009). Promissory notes: From crisis to commons. En http://www.midnightnotes.org/Promissory\%20Notes.pdf 
Monbiot, G. (1994). The tragedy of enclosure. Scientific American (270), 159-160. En Monbiot. com, publicado en enero 1, 1994. En http://www.monbiot.com/1994/01/01/thetragedy-of-enclosure/

Observatorio Metropolitano. (2007). Madrid: ¿la suma de todos? Globalización, territorio, desigualdad. Madrid: Traficantes de Sueños.

Observatorio Metropolitano. (2011). La crisis que viene: algunas notas para afrontar esta década. Madrid: Traficantes de Sueños.

Observatorio Metropolitano. (2013). Paisajes devastados después del ciclo inmobiliario: impactos regionales y urbanos de la crisis (pp. 313-354). Madrid: Traficantes de Sueños.

Olson, M. (1965). The logic of collective action. Public goods and the theory of groups. Cambridge, MA: Harvard University Press.

Ostrom, E. (1990). Governing the commons: The evolution of institutions for collective action. Cambridge, Ru: Cambridge University Press.

Parker, P. \& Johansson, M. (2011). The uses and abuses of Elinor Ostrom"s concept of commons in urban theorizing. Trabajo presentado en International Conference of the European Urban Research Association (EURA), 23-25 junio 2011, Copenhague.

Pasquinelli, M. (2008). Animal spirits: A bestiary of the commons. Rotterdam: NAi Publishers.

Porter, L., Lombard, M., Huxley, M., Ingin, A. K., Islam, T., Briggs, J., Rukmana, D., Devlin, R. \& Watsoi, V. (2011). Informality, the commons and the paradoxes for planning: Conceptsand debatesfor informalityand planning self-made cities: ordinary informality? Planning Theory \& Practice, 12(1), 115-153. doi: 10.1080/14649357.2011.545626

Post, J. \& Baud, I. (2003). Between markets and partnerships: urban solid waste management and contributions to sustainable development? Global Built Environment Review, 3(1), 46-65. En http://dare.uva.nl/document/2/33949

Roggero, G. (2010). Five theses on the common. Rethinking Marxism, 22(3), 357-373. doi: 10.1080/08935696.2010.490369

Saegert, S. \& Benítez, L. (2005). Limited equity housing cooperatives: Defining a niche in the low income housing market. Journal of Planning Literature, 19(4):427-239. doi: $10.1177 / 0885412204274169$

Salingaros, N. A. (2010). P2P Urbanism. [Versión borrador 3.0. Por publicarse en 2011, por Umbau-Verlag, Solingen, en colaboración con la Fundación Peer to Peer]. doi: 10.13140/2.1.1162.2401 Selsky, J. W. \& Memon, P. A. (1997). Urban port development conflicts: towards managing an amenity commons. Urban Policy and Research, 15(4), 259-268. doi: 10.1080/08111149708551371

Serlin, D. \& Chazkel, A. (Eds.). (2010). New approaches to enclosures. Special Issue of Radical History Review (109). Durham, NC: Duke University Press.

Sheldon, J. L. (2010). Community gardens: best practices across urban America. Tesis doctoral, California State University, Sacramento. En http://www.csus.edu/ppa/thesis-project/ bank/2010/Sheldon.pdf

Smith, N. (2002). New globalism, new urbanism: Gentrification as global urban strategy. Antipode, 34(3), 427-450. doi: 10.1111/1467-8330.00249

Sofoulis, Z. \& Williams, C. (2008). From pushing atoms to growing networks: Cultural innovation and co-evolution in urban water conservation. Social Alternatives, 27(3), $50-57$. 
Spronk, S. \& Webber, J. R. (2007). Struggles against accumulation by dispossession in Bolivia The political economy of natural resource contention. Latin American Perspectives, 34(2), 31-47. doi: 10.1177/0094582X06298748

Stavrides, S. (2011, borrador). Brazilian urban movements "re-inventing" the city as collectively produced "common". [No publicado].

Stavrides, S. (2012). Squares in movement. South Atlantic Quarterly, 111(3), 585-596. doi: $10.1215 / 00382876-1596308$

Svendsen, E. \& Campbell, L. K. (2008). Urban ecological stewardship: Understanding the structure, function and network of community-based urban land management. Cities and the Environment (CATE), 1(1), 1-32. En http://www.nrs.fs.fed.us/pubs/jrnl/2008/ nrs_2008_svendsen_001.pdf

Swyngedouw, E. (2014). Where is the political? Insurgent mobilizationsand theincipient"return of the political". Space and Polity, 18(2), 122-136. doi: 10.1080/13562576.2013.879774

Theodore, N., Peck, J. \& Brenner, N. (2009). Urbanismo neoliberal: la ciudad y el imperio de los mercados. Temas Sociales, (66). Santiago: sur Corporación de Estudios Sociales y Educación. En http://www.sitiosur.cl/r.php?id=898

Vasudevan, A., McFarlane, C. \& Jeffrey, A. (2008). Spaces of enclosure. Geoforum, 39(5), 1641-1646. http://dx.doi.org/10.1016/j.geoforum.2008.03.001

Vercellone, C. (2009). Crisis de la ley del valor y devenir renta de la ganancia. Apuntes sobre la crisis sistémica del capitalismo cognitivo. En S. Mezzadra (Comp.), La gran crisis de la economía global (pp. 63-98). Madrid: Traficantes de Sueños.

Wagenaar, P. \& Soeparman, S. (2004). Coping with the dilemma of common pool information resourcing: Integrating information domains in the Dutch police. Information Polity, 9(3/4):181-192.

Wutich, A. (2009). Water scarcity and the sustainability of a common pool resource institution in the urban Andes. Human Ecology, 37(2), 179-192. doi: 10.1007/ s10745-009-9227-4

Žižek, S. (2009). How to begin from the beginning. New Left Review, (57), 43-56. En http:// newleftreview.org/II/57/slavoj-zizek-how-to-begin-from-the-beginning 
